\title{
INFLUENCE D'UNE FINE SÉDIMENTATION DANS UN CANAL EXPÉRIMENTAL SUR LA DENSITÉ DU MACROBENTHOS, SA COMPOSITION ET SA CONSOMMATION PAR DES SALMONIDES
}

\author{
A. NEVEU *
}

\section{RESUME}

Les relations entre la qualité du substrat, la densité benthique et la nourriture de Salmonides ont été etudiées dans un ruisseau expérimental pour déterminer les effets d'un apport limoneux. L'eau était captée dans une rivière dont le brassin subit une forte érosion. Au début la composition du benthos etait la même dans les deux systèmes, mais avec des stocks plus èlevés dans le ruisseau artificiel, en relation avec l'absence de poisson et la régulation du déhit. La sédimentation commença avec les crues de la rivière. les cailloux furent progressivement colmatés par la boue. La diversité et la biomasse du benthos sont alors réduites par ce changement de la qualité du substrat. La consommation du poisson, liée à la densité benthique, est plus basse dans les zones colmatées. Ces changements fauniques affectent aussi le choix, les contenus stomacaux sont plus diversifiés lorsque la nourriture est rare.

- IN.R.A., Laboratoire d'Ecologie hydrobiologique - E.N.A., 65 rue de Saint Brieuc, 35042 RENNES CEDEX. 


\section{SUMMARY}

The relationship between substrat quality, benthos density and food level for Salmonid was studied in an artificial stream to determine the effects of sitt deposition. Water was caught from a natural river with eroded tributaries. At the beginning invertebrate fauna has the same composition in the two systems, but benthic stocks were higher in artificial channel, because there was no fish predation and a regulated fiow. Sedimentation has begun with river floods, gravels were progressively clogged with silt. Benthos diversity and standing crop are reduced by this change of substrate quality. Fish predation is correlated with food density and consumption rate is lower in stream in stream sections with. mud. Change in food disponibility affect also fish selectivity, stomach contents have more diversified items when benthos is scarce.

\section{INTRODUCTION}

De nombreuses études montrent que toute sédimentation est néfaste au développement des Salmonides (PETERS, 1967; HALL, LANTZ, 1968; LANTZ 1971 ; RITCHIE, 1972 ; CLOERN, 1976). Or les pollutions physiques (ou mécaniques) se développent de plus en plus avec les extractions de graviers, les chantiers autoroutiers, le déboisement, certaines pratiques agricoles (monoculture, surpâturage, travaux connexes au remembrement), etc... Tous ces facteurs ont pour conséquence un apport massif de matières en suspension dans les cours d'eau récepteurs.

Cette action sur les Salmonides peut être directe, au niveau de la fertilité réelle (colmatage des frayères), mais aussi indirecte, au niveau du benthos, donc de la nourriture disponible (ROSENBERG, SNOW, 1975; LUEDTKE, BRUSVEN, 1976 ; CLAVEL et al, 1978). Toute réduction alimentaire a en effet pour conséquence un ralentissement de croissance d'autant plus important que la température de l'eau est élevée (ELLIOTT, 1975). Dans le cas des Salmonides cette réduction exacerbe les antagonistes territoriaux et la densité en résidents diminue (SYMONS, 1968, 1971; SLANEY, NORTHCOTE, 1974).

Le but de ce travail est donc de présenter simultanement les variations du benthos, de la quantite de sédiments et de la consommation de différents Salmonides (S. trutta, S. gairdneri, S. salar).

\section{TECHNIQUES ET METHODES}

Les expériences se sont déroulées dans des canaux en béton de $200 \mathrm{~m}$ sur $1,20 \mathrm{~m}$ ayant une pente de $0,5 \%$, dont le fond est recouvert de deux couches de cailloux anguleux de 8 à $12 \mathrm{~cm}$ de diamètre. Ces cailloux laissent entre eux des espaces importants, colonisables par les invertébrés. Afin de simuler les conditions naturelles, une rive de $0,20 \mathrm{~m}$ est recouverte de plantes hygrophiles diverses, support de faune exogène. Des herbiers à renoncules aquatiques, régulierement répartis, permettent une diversification de l'habitat.

L'alimentation en eau est effectuée à partir d'un petit fleuve côtier de l'Ouest des Pyrénées : la Nivelle. Cette région présente des risques d'érosion hydrique parmi les plus éleves de France, en relation avec lagressivité des pluies évaluée par l'indice de WISCHMEIER (PIHAN, 1978). Le bassin de ce cours d'eau 
reçoit d'importantes précipitations $(1700 \mathrm{~mm})$, qui, compte tenu de sa faible taille et de la forte pente, provoquent des crues nombreuses et violentes. L'eau est alors très chargée en limons provenant de la décomposition et du lessivage. des terrains schisteux non protégés par une couverture végétale suffisante. Celleci s'est dégradée depuis le déboisement des pentes et le développement de l'élevage du mouton sur des pâtures que l'on brù'e au printemps. Ces limons, très fins, d'origine argileuse, rendent l'eau trouble les deux tiers de l'année (LAPCHIN, NEVEU, sous presse).

L'installation d'une pisciculture expérimentale sur ce type de cours d'eau pose de nombreux problèmes. En effet, la régularisation du débit dans les installations d'élevage déclenche la sédimentation d'une partie des argiles. Le colmatage est accéléré par un développement algual important, consécutif à la température élevée de l'eau associée aux apports minéraux adsorbés sur les boues (LAPCHIN. NEVEU, sous presse). Ces algues constituent un véritable piège à sédiments (BISSON, DAVIS, 1976).

Les prélèvements d'invertèbrés ont été effectués à l'aide d'un filet de Surber de $0,1 \mathrm{~m}^{2}$ à mailles de $0,25 \mathrm{~mm}$, ceux de boue à l'aide d'un filet à mailles de $0.07 \mathrm{~mm}$; les cailloux étaient remués dans les mêmes conditions, soit pour déloger les invertébrés, soit la boue. Cette méthode de mesure est seulement une approche de la véritable quantité de sédiment; elle donne cependant une idée du taux de colmatage. Le volume de boue est mesuré après sédimentation de 2 heures dans une éprouvette graduée

Les prélèvements stomacaux ont toujours été effectués au cours de la même période du cycle d'alimentation, entre 15 et $16 \mathrm{~h}$. par abattage du poisson et fixation au formol $5 \%$. Après analyse des composants, ceux-ci ont été séchés 24 heures à $60^{\circ} \mathrm{C}$, puis pesés

\section{RESULTATS}

La mise en eau fut effectuée en février 1976 avec un débit élevé (de 40 à $60 \mathrm{l} / \mathrm{sec}$ ); début juin un contrōle ne permet pas de mettre en évidence une différence significative de la densité du benthos entre l'amont et l'aval, malgré un début de sédimentation et de colmataqe des cailloux les plus profonds. Faute d'un débit estival suffisant de la Nivelle, celui du canal expérimental est alors rapporté à $10-15 \mathrm{l} / \mathrm{sec}$. En septembre, à la suite des orages estivaux dont l'érosivité est maximum (PIHAN. 1978). le colmatane est bien visible. L'arrivée des pluies d'automne permet d'augmenter le débit à $20-30 \mathrm{l} / \mathrm{sec}$. ce qui correspond à une vitesse moyenne du courant de $0,30 \mathrm{~m} / \mathrm{sec}$. le long du canal; le facies est donc nettement lotique d'amont en aval.

\section{1) Expérience no 1 : décembre 1976 - Truites communes}

Trois semaines après l'introduction des poissons, issus de pisciculture, la rigole est divisée en 4 parties égales par des repères : de l'amont vers l'aval. les secteurs 1, 2, 3 et 4 . Au centre de chacune de ces zones sont prélevés: 1 échantillon benthique $\left(S=0,1 \mathrm{~m}^{2}\right)$ et 2 échantillons de boue $\left(S=0.2 \mathrm{~m}^{2}\right)$, puis les truites (8 à 11 individus) sont capturées à l'électricité dans chaque secteur.

\section{A. LE SUBSTRAT}

Les cailloux sont de plus en plus colmatés de l'amont vers l'aval avec 
des volumes de boue de : 17, 37, $76.122\left(\mathrm{~cm}^{3} / 0,2 \mathrm{~m}^{2}\right)$, soit environ 7 fois plus de dépôts en fin de canal. Ce dépôt de limons se produit malgré l'absence de tous obstacles à l'écoulement (grilles, barrages,...), la lame d'eau reste sensiblement constante d'amont en aval, avec en particulier la même vitesse moyenne $(0,30 \mathrm{~m} / \mathrm{sec}$.). Or, à partir d'observations effectuées dans la rivière, il semblerait qu'une vitesse d'au moins $0,60-0,70 \mathrm{~m} / \mathrm{sec}$. soit nécessaire pour empêcher la sédimentation, sans oublier l'effet des crues.

\section{B. LE BENTHOS}

Les espèces des diverses familles d'invertébrés sont rarement différentes de celles de la Nivelle, étudièes en détail par NEVEU. LAPCHIN. VIGNES (1979).

La composition numérique ne présente pas de grandes variations; l'importance relative des Chironomidæ augmente vers l'aval, celle des Elminthidæ diminue (TAB. 1). Cette relative stabilité laisse donc supposer que si la densité totale change, ceci se fait d'une manière équitable pour la plupart des groupes, les Gammaridæ étant nettement dominants. Les larves de Simuliidæ sont rares, ce sont essentiellement des Wilhelmia qui se fixent préférentiellement sur les tiges de renoncules, mais aussi sur les cailloux lorsque ceux-ci restent propres (NEVEU, LAPCHIN, 1979)

L'indice de diversité de Shannon peut s'appliquer aux familles :

$$
D=-\underset{1}{1} P_{i} \log _{2} P_{i}
$$

$i=$ nombre de familles présentes $-P_{i}=$ fréquence de la famille $i$

II atteint les valeurs absolues suivantes $1,77-2,02-1,92-2,20$ pour les secteurs 1.2. 3 et 4 . Ces variations sont donc irrégulières

L'examen des variations de densité numérique des principaux taxons montre : une forte diminution amont - aval des Gammaridæ (Echinogammarus berilloni Pink.), des Ancylidæ (Ancylus fluviatilis Mül.), des Elminthidæ, des Ecdyonuridæ (Rhithrogena semicolorata Cur.), une plus faible réduction des Polycentropidæe (Polycentropus flavomaculatus $P i c$.) ,une certaine stabilité des Bætidæ (surtout $B$. rhodani Pic.). Par contre il y a une augmentation très nette des Chironomidæ, les formes rouges, plus résistantes au manque d'oxygène, n'étant présentes qu'en aval (Fig. 1 A, B, C). 
Tableau 1 : Variations longitudinales de la composition numérique du benthos en décembre 1976

\begin{tabular}{|c|c|c|c|c|}
\hline \multirow{2}{*}{ FAMILLES (pour cent) } & \multicolumn{4}{|c|}{ Secteurs } \\
\hline & 1 & 2 & 3 & 4 \\
\hline Ephémèroptères & & & & \\
\hline $\begin{array}{l}\text { Bætidæ } \\
\text { Ecdyonuridæ }\end{array}$ & $\begin{array}{l}3 \\
1,3\end{array}$ & $\begin{array}{l}3 \\
1,2\end{array}$ & $\begin{array}{l}6,5 \\
0,4\end{array}$ & $\frac{11,5}{-}$ \\
\hline Plécoptères & & & & \\
\hline $\begin{array}{l}\text { Leuctridæ } \\
\text { Trichoptères }\end{array}$ & - & 0,2 & - & 0,6 \\
\hline $\begin{array}{l}\text { Hydropsychidæ } \\
\text { Polycentropidæ } \\
\text { Goridæ } \\
\text { Philopotamidæ } \\
\text { Lepidostomatidæ }\end{array}$ & $\begin{array}{l}0,4 \\
2 \\
- \\
-\end{array}$ & $\begin{array}{l}0,5 \\
5,8 \\
0,2 \\
0,2 \\
0,7\end{array}$ & $\begin{array}{l}0,7 \\
6,5 \\
- \\
-\end{array}$ & $\begin{array}{l}0,6 \\
5,7 \\
- \\
- \\
-\end{array}$ \\
\hline Coléoptères & & & & \\
\hline $\begin{aligned} \text { Elminthidæ } & L \\
& \mathrm{Ad}\end{aligned}$ & $\begin{array}{c}19,5 \\
3\end{array}$ & $\begin{array}{l}7,4 \\
0,5\end{array}$ & $\frac{4,3}{-}$ & $\stackrel{2,3}{-}$ \\
\hline Mégaloptères & & & & \\
\hline $\begin{array}{l}\qquad \text { Sialidæ } \\
\text { Diptères }\end{array}$ & - & 0,7 & - & - \\
\hline $\begin{array}{l}\text { Chironomidæ } \\
\text { Simuliidæ } \\
\text { Tabanidæ } \\
\text { Limoniidæ } \\
\text { Athericidæ }\end{array}$ & $\begin{array}{l}0,3 \\
0,1 \\
0,2 \\
0,3 \\
0,1\end{array}$ & $\begin{array}{l}2,1 \\
0,2 \\
\overline{0}, 2 \\
-\end{array}$ & $\begin{array}{l}3,9 \\
1,1 \\
0,4 \\
-\end{array}$ & $\begin{array}{r}10,9 \\
1,1 \\
1,1 \\
- \\
-\end{array}$ \\
\hline Amphipodes & & & & \\
\hline $\begin{array}{l}\text { Gammaridæ } \\
\text { Mollusques }\end{array}$ & 58,3 & 57,8 & 64,5 & 55,7 \\
\hline $\begin{array}{l}\text { Ancylidæ } \\
\text { Physidæ }\end{array}$ & $\begin{array}{r}11,4 \\
0,3\end{array}$ & $\underline{19,3}$ & $\begin{array}{l}10 \\
1,4\end{array}$ & $\begin{array}{l}6,9 \\
3,4\end{array}$ \\
\hline Hydracariens & 0,2 & - & 0,4 & - \\
\hline
\end{tabular}


$-109-$

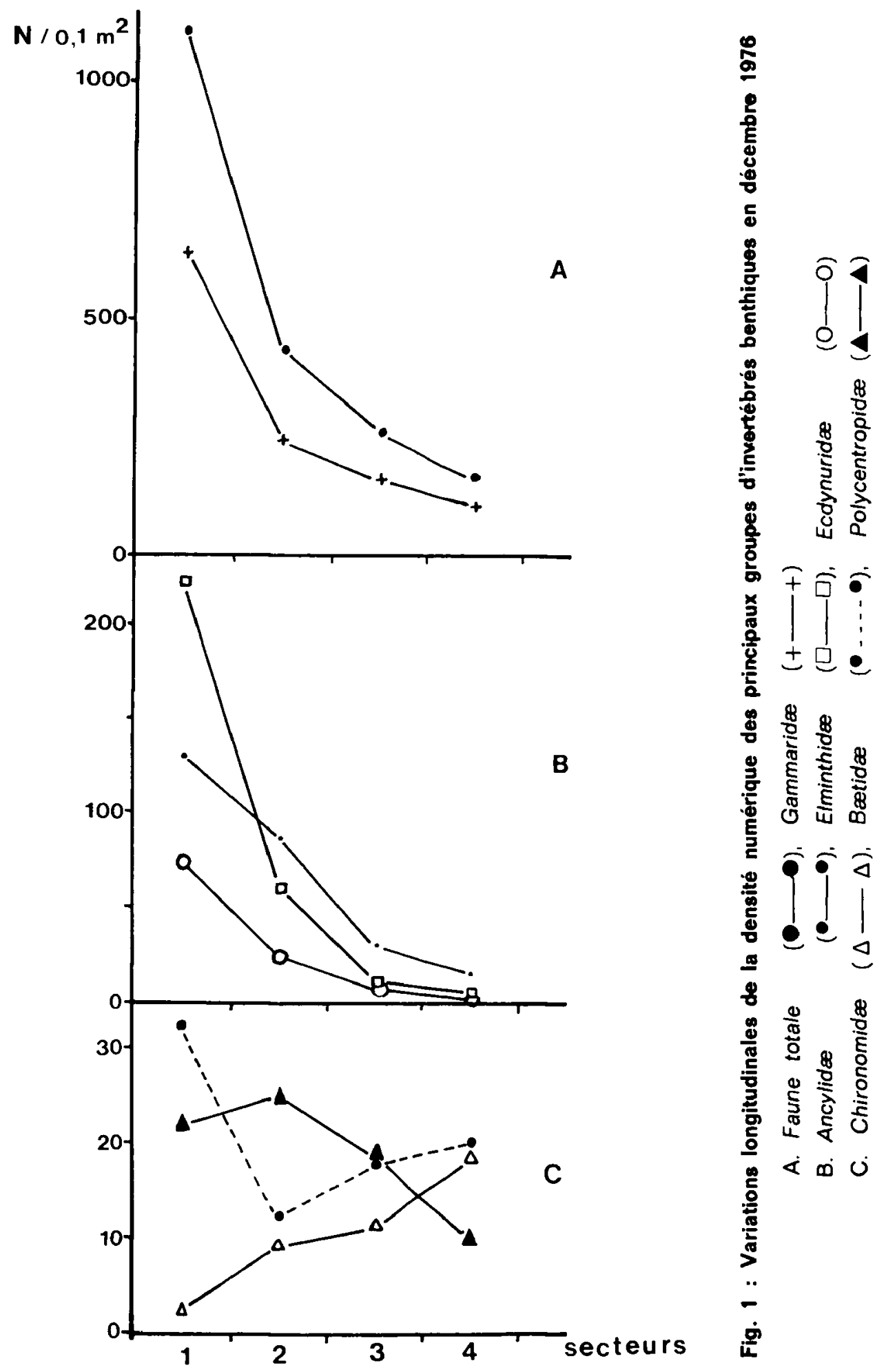


La densité numérique du benthos dans la partie la plus basse n'est plus que $16 \%$ de celle de la partie la plus amont (Fig. 1 A). Cette diminution des stocks benthiques est liée à l'accroissement du taux de boue (Fig. 2). Ces courbes sont des portions d'hyperboles : la densité numérique $N=7214,32 \times{ }^{-0,91}$ ( $N$ pour $0.1 \mathrm{~m}^{2}$. X quantité de boue $\mathrm{cm}^{3} / 0,1 \mathrm{~m}^{2}$ ) avec $r=0,990$ pour $n=4$, la densité pondérale $B=17870,30 \times{ }^{-0.56}\left(B\right.$ en $\left.\mathrm{mg} / 0,1 \mathrm{~m}^{2}\right)$ avec $r=0,997$ pour les mèmes couples.

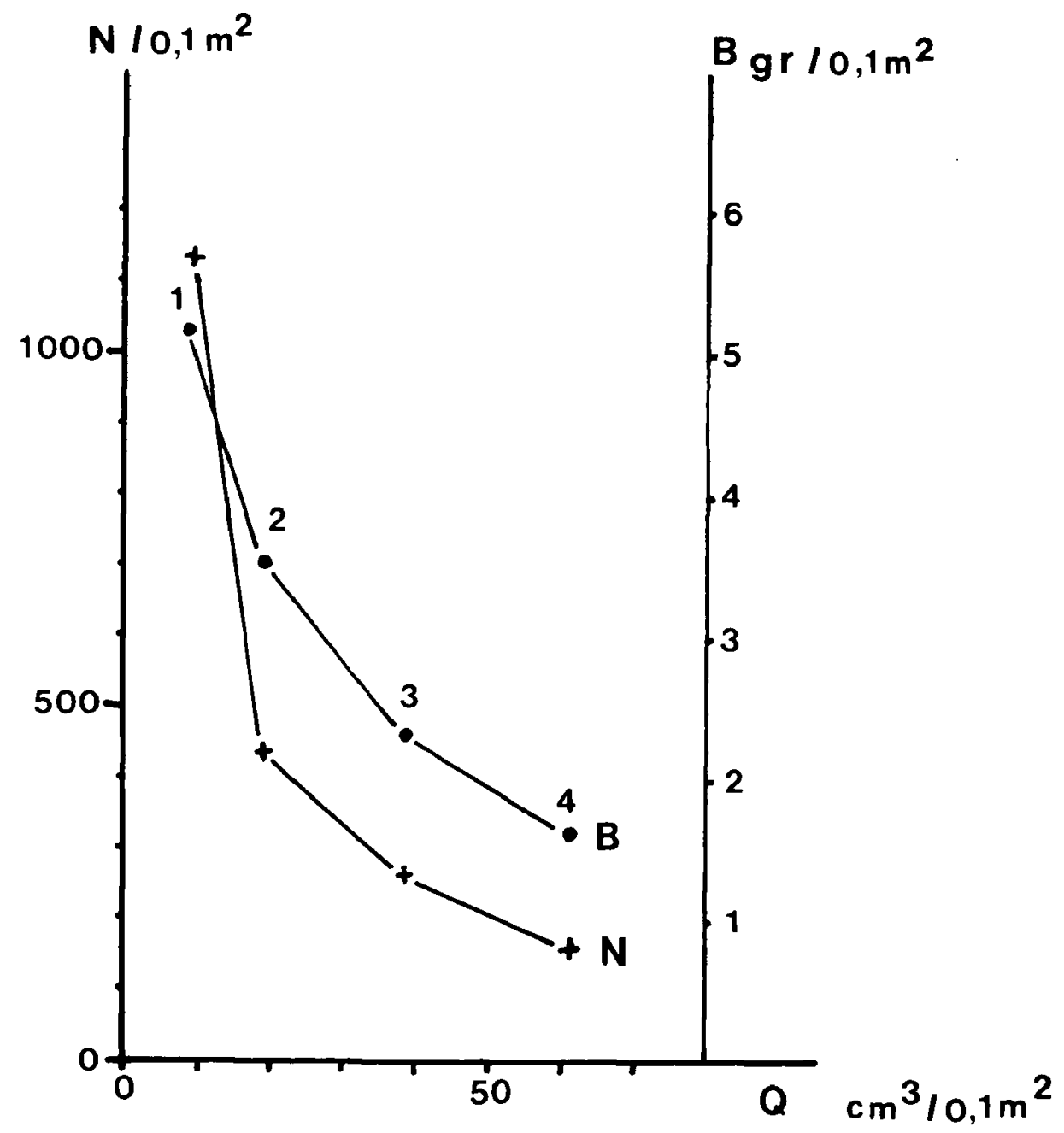

Fig. 2 : Relations entre la densité numérique (N), la biomasse (B) du benthos et la quantité de boue $(Q)$ déposée sur les cailloux des différents secteurs en décembre 1976.

Il est remarquable que les stocks benthiques en aval soient à peu près les mêmes que ceux de la Nivelle pour cette époque (NEVEU, LAPCHIN, VIGNES, 1979). Par contre ceux d'amont sont nettement supérieurs, même au maximum an- 
nuel de la Nivelle qui est d'environ $25 \mathrm{gr} / \mathrm{m}^{2}$ en juin. Ceci peut s'expliquer par l'absence de poissons pendant de nombreux mois, la colonisation d'un nouveau milieu, la diversité de l'habitat et surtout la régularisation du débit qui évite l'arrachement des animaux par les crues. Les stocks de décembre sont cependant beaucoup plus faibles que ceux observés au cours du contrôle de juin 1976, qui étaient de $81,8 \mathrm{gr} / \mathrm{m}^{2}$, grâce à l'abondance des Ancylidæ, des Gammaridæ, des Ephéméroptères et à la propreté du milieu consécutif au maintien d'un fort débit (40 à $60 \mathrm{l} / \mathrm{sec})$.

\section{L'ALIMENTATION DES TRUITES}

Les truites utilisées avaient une taille moyenne de $14,2 \mathrm{~cm}(\sigma=0,8)$ et un poids moyen de $38,1 \mathrm{gr}(\sigma=5,4)$.

La composition numérique du contenu stomacal présente des variations entre les différents secteurs (TAB. 2). Malgré la présence d"herbiers à renoncules sur toute la rigole, l'importance relative des larves de Simuliidæ diminue d'amont en aval, celle des Mollusques, des Trichoptères augmente, celle des Gammaridæ et des Bætidæ est variable. La faune terrestre tend à augmenter vers l'aval en relation avec les apports issus des plantes rivulaires, mais ils restent faibles. Il faut noter la présence de nymphes de Simulies avec leurs cocons. ceci laisse supposer un "broutage" de ces animaux sur les renoncules.

Il est possible de préciser le choix des truites par l'application de l'indice de sélectivité d'IVLEV (1961) (Fig. 3)

$$
E=\frac{r_{i}-p_{i}}{r_{i}+p_{i}}
$$

$r_{i}=$ fréquence de la proie dans la ration, $p_{i}=$ fréquence de la proie dans son milieu. Le choix des truites envers les Simuliidæ diminue vers l'aval, mais reste toujours positif; il en est de même des Bætidæ. Par contre, celui des Mollusques et des Trichoptères augmente. En amont où les proies préférées sont abondantes, elles sont fortement consommées, en aval leur diminution entraine un report sur des groupes normalement peu sélectionnés. c'est en narticulier le cas des Ancylidæ malgré leur faible densité. Cette attractivité des Mollusques en aval est accentuée par le développement des Physidæe et leur accessibilité permanente. Par contre, les Chironomidæe, malaré l'arıamentation de leurs ponulations, restent peu accessibles. Par rapoort à lelır abondance relative les Gammaridæ ne sont pas particulièrement choisis, mais ce choix reste équitable d'amont en aval.

Si en haut du canal 8 familles d'invertébrés benthiques sont consommées, en bas il y en a 13; face à une réduction des stocks, il y a diversification des proies capturées. L'indice de diversité des contenus stomacaux suit cette évolution amont-aval : 1,61 - 1,24-2,10-2,83.

La quantité de proies ingérées a tendance aussi à diminuer, mais ce changement est beaucoup plus net au niveau du poids sec du contenu stomacal (Fig. 4A). L'intensité de la consommation est liée positivement à la densité du benthos (Fig. 4 B) donc négativement au taux de sédiments (Fig.2). 
Tableau 2 : Variations longitudinales de la composition numérique des contenus stomacaux de truites communes (S. trutta) en décembre 1976

\begin{tabular}{|c|c|c|c|c|}
\hline \multirow{2}{*}{ FAMILLES (pour cent) } & \multicolumn{4}{|c|}{ Secteurs } \\
\hline & 1 & 2 & 3 & 4 \\
\hline \multicolumn{5}{|l|}{ Ephémèroptères } \\
\hline Bætidæ & 10,5 & 3,1 & 18,2 & 6.4 \\
\hline \multicolumn{5}{|l|}{ Plécoptères } \\
\hline $\begin{array}{l}\text { Nemuridæ } \\
\text { Tæniopterygidæ }\end{array}$ & $\stackrel{0,6}{-}$ & $\overline{0.5}$ & - & - \\
\hline \multicolumn{5}{|l|}{ Trichoptères } \\
\hline Rhyacophilidæ & 0,3 & - & 5,3 & 5,5 \\
\hline Hydropsychidæ & - & 0,6 & & 0,9 \\
\hline Hydroptilidæe & 1,4 & 0,5 & 1,1 & 3,6 \\
\hline Polycentropidæ & - & - & 一 & 0,9 \\
\hline Nymphes sp. & - & - & 0,8 & - \\
\hline \multicolumn{5}{|l|}{ Mégaloptères } \\
\hline Sialidæ & - & 0,2 & - & - \\
\hline \multicolumn{5}{|l|}{ Diptères } \\
\hline Simuliidæ L & 45,5 & 61,9 & 42,2 & 16,4 \\
\hline $\mathrm{Ny}$ & 0,9 & 3,6 & 2,3 & 0,9 \\
\hline$A d$ & - & - & 0,8 & - \\
\hline Chironomidæ $\mathrm{L}$ & 一 & 0,2 & - & 0,9 \\
\hline $\mathrm{Ny}$ & - & - & - & 0.9 \\
\hline $\mathrm{Ad}$ & - & 0.2 & 0,4 & - \\
\hline \multicolumn{5}{|l|}{ Amphipodes } \\
\hline Gammaridæ & 39,8 & 27,9 & 20,9 & 31,8 \\
\hline \multicolumn{5}{|l|}{ Isopodes } \\
\hline Asellidæ & 0.3 & - & 0,4 & 0,9 \\
\hline \multicolumn{5}{|l|}{ Mollusques } \\
\hline Ancylidæ & 0,6 & - & 2,3 & 11,8 \\
\hline Hydrobiidæ & - & - & 1,1 & 0,9 \\
\hline Physidæ & - & - & 0,8 & 12,7 \\
\hline Sphæriidæ & - & 一 & - & 0.9 \\
\hline Divers terrestres & 0,3 & 1,1 & 3 & 4,5 \\
\hline
\end{tabular}




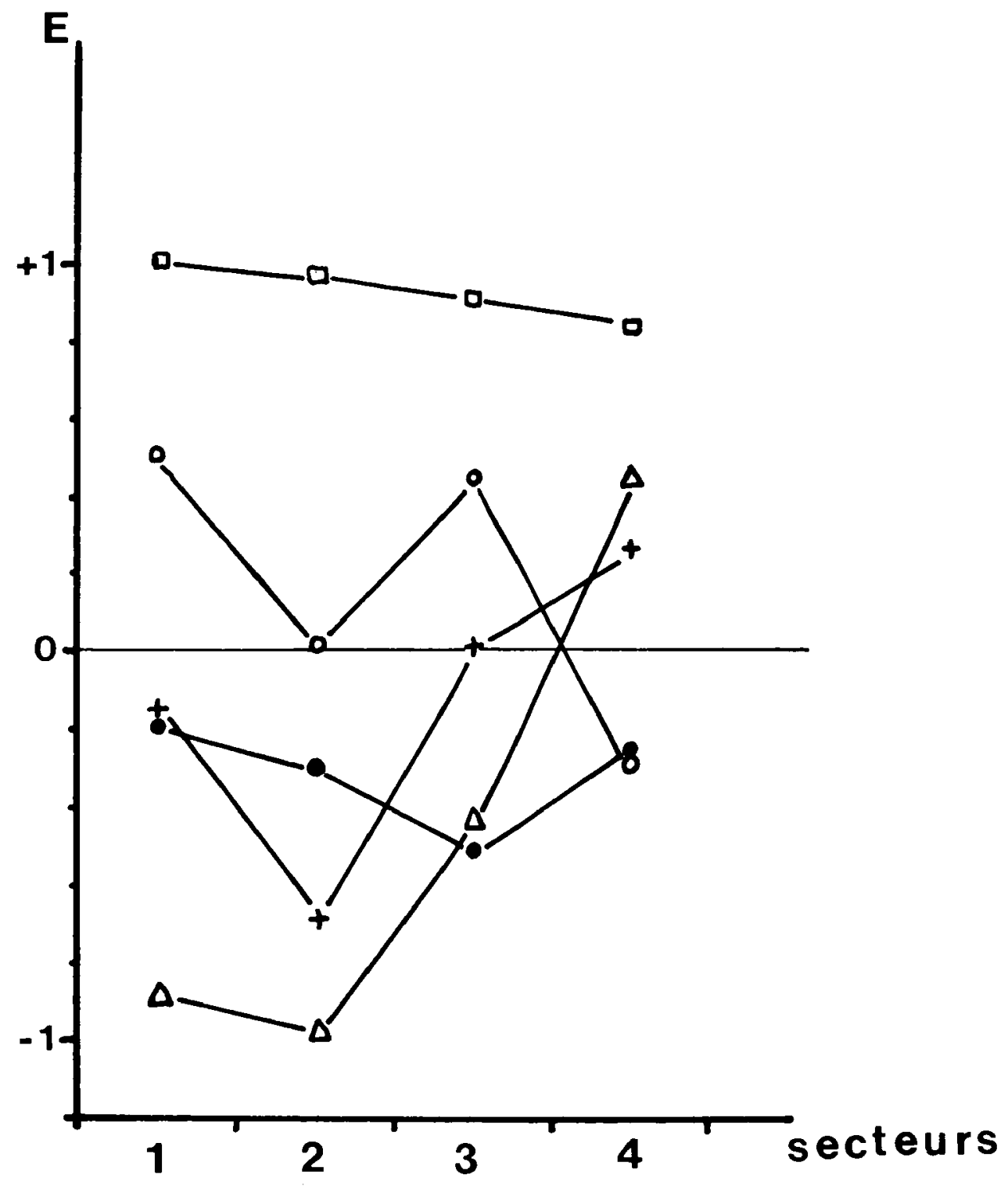

Fig. 3 : Variations longitudinales de l'indice de sélectivité d'IVLEV (E) appliqué aux principaux composants des contenus stomacaux de truites communes (décembre 1976).

$\begin{array}{llll}\text { Mollusques } & (\Delta-\Delta), & \text { Trichoptères } & (+-+) \\ \text { Simuliidæ } & (\square-\square), & \text { Gammaridæ } & (\bullet-\bullet) \\ \text { Bætidæ } & (\mathrm{O}-0) . & \end{array}$ 
P mg

$\mathrm{K}$
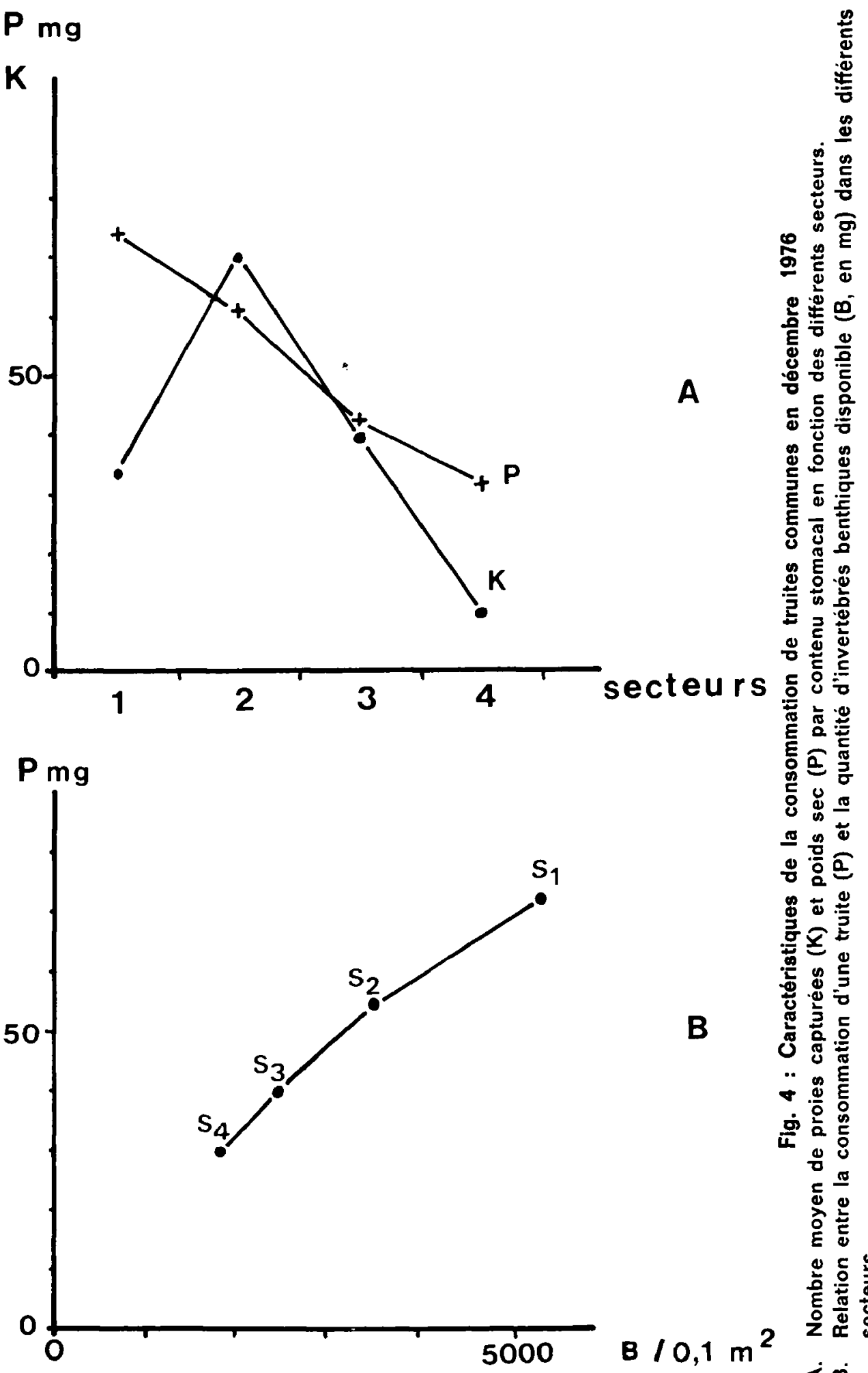

हू。

氞票

\&

क ष

흠도

另 志

兽全

B

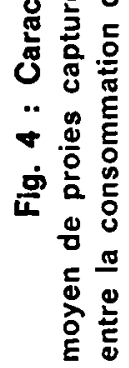

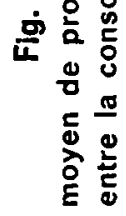

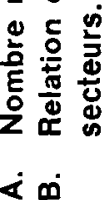




\section{2) Expérience n॰ 2 : février 1977 - Truites communes}

Le milieu est à nouveau empoissonné avec des truites communes de picciculture 3 semaines avant l'étude. Au moment de leur capture, ces truites mesuraient $15,2 \mathrm{~cm}(\sigma=0,6)$ avec un poids de $42,7 \mathrm{gr}(\sigma=6,6)$. Au niveau du benthos, seul le groupe des Gammaridæ est trié pour servir d'indicateur des variations des stocks de nourriture disponible, compte tenu des particularités de son cycle de développement.

Les résultats sont comparables, la composition des contenus stomacaux reste assez stable d'amont en aval, avec cependant une augmentation plus forte des Mollusques (TAB. 3). Les principales différences avec décembre sont : une baisse des larves de Simuliidæ compensèe par une consommation d'adultes émergeant et de nombreux subimagos de Bæetidæ. L'indice de diversité varie peu : de 2,12 en amont à 2,42 en aval.

Si le nombre de proies par estomac ne change guère, le poids sec du contenu diminue de l'amont vers l'aval, il en est de même de la densité numérique des Gammaridæe en relation avec l'augmentation du colmatage (TAB 4).

\section{3) Expérience n॰ 3 : mai 1977 - Saumon atlantique}

Le colmatage ne fait qu'augmenter avec le temps, si bien que les cailloux ne sont plus visibles dans l'extrême partie aval. Le filet de Surber se colmate immédiatement et les prélèvements tant de faune que de boue sont aléatoires; ils sont donc abandonnés. Ce colmatage rehausse légèrement le fond vers l'aval, ce qui contribue à diminuer la pente et ne peut qu'accélérer le processus. Seuls les contenus stomacaux sont étudiés.

Des juvéniles de Saumon atlantique, issus de pisciculture, sont mis en place 15 jours avant l'échantillonnage, après élimination de tous les autres poissons. Les estomacs de 10 individus sont prélevés dans la zone 1 et 10 dans la zone 4. La taille de ces poissons est en moyenne de $8,2 \mathrm{~cm}(J=0,9)$ et le poids de $6,9 \mathrm{gr}(\sigma=2,3)$.

La composition des contenus stomacaux reste toujours assez proche dans les deux secteurs (TAB 5). On notera l'importance relative des Bætidæ, des Chironomidæ, une baisse des Gammaridæ par rapport aux truites communes, mais cela peut être lié à la saison et aux cycles de développement de ces espèces (NEVEU, LAPCHIN, VIGNES, 1979). La diversité des proies baisse en aval puisque l'indice de Shannon passe de 2,21 à 1,78 pour les familles benthiques. Quant à l'intensité de la consommation elle est aussi fortement affectée.

\section{4) Expérience no 4 : juin 1977 - Truites Arc en ciel}

Même type d'expérience simplifiée que la précédente avec des truites arcen-ciel de $7,3 \mathrm{~cm}(\sigma=0,5)$ et de $5,2 \mathrm{gr}(\sigma=1,7)$, issues de pisciculture et placées 15 jours avant dans la rigole, après élimination des autres espèces.

Avec le temps, la hauteur de boue augmente moins que sa cohésion, elle devient de plus en plus ferme. Cette stabilité favorise le développement d'algues filamenteuses et d'herbiers à renoncules.

Les Gammaridæ sont à nouveau la nourriture de base, ils baissent légèrement en aval (TAB. 6). Les Chironomidæ, les adultes de Simuliidæ et les Polycentropidæ prennent de l'importance en aval. L'indice de diversité passe de 1,27 
Tableau 3 : Variations longitudinales de la composition numérique des contenus stomacaux de truites communes ( $S$. trutta) en février 1977

\begin{tabular}{|c|c|c|c|c|}
\hline \multirow{2}{*}{ FAMILLES (pour cent) } & \multicolumn{4}{|c|}{ Secteurs } \\
\hline & 1 & 2 & 3 & 4 \\
\hline Ephémèroptères & & & & \\
\hline $\begin{array}{r}\text { Bætidæ L } \\
\text { Subi. }\end{array}$ & $\begin{array}{r}2,5 \\
36,6\end{array}$ & $\begin{array}{r}0,7 \\
12,5\end{array}$ & $\begin{array}{c}0,6 \\
16\end{array}$ & $\begin{array}{l}6,9 \\
6,2\end{array}$ \\
\hline Trichoptères & & & & \\
\hline $\begin{array}{l}\text { Polycentropidæ } \\
\text { Nymphes Sp. }\end{array}$ & $\begin{array}{l}0,4 \\
0,8\end{array}$ & 1,4 & $\begin{array}{l}0,6 \\
1,2\end{array}$ & $\overline{-}$ \\
\hline Diptères & & & & \\
\hline $\begin{array}{ll}\text { Simuliidæ } & \mathrm{L} \\
& \mathrm{Ny} \\
& \mathrm{Ad}\end{array}$ & $\begin{array}{r}1,7 \\
2,9 \\
13,9\end{array}$ & $\begin{array}{c}13,2 \\
11,1 \\
9\end{array}$ & $\begin{array}{r}14,1 \\
7,4 \\
5,5\end{array}$ & $\begin{array}{r}2,1 \\
2,1 \\
11,7\end{array}$ \\
\hline $\begin{aligned} & \text { Chironomidæ } \mathrm{L} \\
& \mathrm{Ny} \\
& \mathrm{Ad}\end{aligned}$ & $\frac{0,4}{4,6}$ & $\begin{array}{l}\overline{0,7} \\
1,4\end{array}$ & $\begin{array}{l}\overline{3,7} \\
3,7\end{array}$ & $\begin{array}{l}0,7 \\
2,8 \\
2,1\end{array}$ \\
\hline Mollusques & & & & \\
\hline $\begin{array}{l}\text { Ancylidæ } \\
\text { Physidæ } \\
\text { Hydrobiidæ }\end{array}$ & $\begin{array}{l}2,9 \\
1,2 \\
0,6\end{array}$ & $\begin{array}{r}9 \\
1,4 \\
0,7\end{array}$ & $\begin{array}{l}9,8 \\
1,2 \\
-\end{array}$ & $\begin{array}{r}31,7 \\
3,4 \\
2,1\end{array}$ \\
\hline Amphipodes & & & & \\
\hline Gammaridæe & 29 & 36,8 & 33,1 & 21,4 \\
\hline Hydracariens & 0,6 & 一 & 0,6 & - \\
\hline Divers terrestres & 1,7 & 2,1 & 2,5 & 6,9 \\
\hline
\end{tabular}


Tableau 4 : Relations entre les contenus stomacaux de février 1977, la densité des Gammaridæe et la quantité de boue

\begin{tabular}{|c|c|c|c|c|}
\hline & \multicolumn{4}{|c|}{ Secteurs } \\
\cline { 2 - 5 } & 1 & 2 & 3 & 4 \\
\cline { 2 - 5 } Contenus stomacaux pour 10 truites : & & & & \\
Poids sec (mg) & 953,2 & 806,1 & 602,3 & 617,3 \\
Nombre de proies & 238 & 160 & 163 & 161 \\
$\begin{array}{c}\text { Densité des Gammaridæ } \\
\text { sur 0,2 } \mathrm{m}^{2}\end{array}$ & 281 & 296 & 130 & 32 \\
$\begin{array}{c}\text { Densité de la boue } \\
\mathrm{cm}^{3} / 0,2 \mathrm{~m}^{2}\end{array}$ & 54 & 48 & 124 & 195 \\
\hline
\end{tabular}

Tableau 5 : Variations qualitatives et quantitatives des contenus stomacaux de juvéniles de saumons atlantiques ( $S$. salar) sous l'influence du colmatage du substrat en mai 1977

\begin{tabular}{|c|c|c|}
\hline \multirow{2}{*}{ FAMILLES (pour cent) } & \multicolumn{2}{|c|}{ Secteurs } \\
\hline & 1 & 4 \\
\hline Gammaridæ & 18,8 & 19 \\
\hline $\begin{array}{ll}\text { Simuliidæ } & \mathrm{L} \\
& \mathrm{Ny}\end{array}$ & $\begin{array}{l}7,3 \\
1,8\end{array}$ & $\overline{-}$ \\
\hline 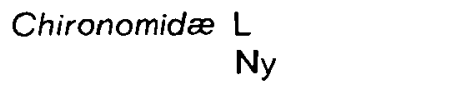 & $\begin{array}{r}13,3 \\
1,2\end{array}$ & $\begin{array}{r}11,9 \\
2,4\end{array}$ \\
\hline Bætidæ & 47,9 & 57.1 \\
\hline Ephemerellidæ & 3 & 2,4 \\
\hline Polycentropidæ & - & 4,8 \\
\hline Rhyacophilidæ & 3,6 & - \\
\hline Hydroptilidæ & 0,6 & 2.4 \\
\hline Nymphes Trichoptères & 1,2 & 一 \\
\hline Leutridæ & 0,6 & - \\
\hline Ancylidæ & 0,6 & - \\
\hline $\begin{array}{l}\text { Poids secs (mg) } \\
\text { pour } 10 \text { saumons }\end{array}$ & 278,1 & 181 \\
\hline Proies/10 saumons & 206 & 105 \\
\hline
\end{tabular}


Tableau 6 : Variations qualitatives et quantitatives des contenus stomacaux de truites arc-en-ciel (S. gairdneri) sous l'influence du colmatage du substrat en juin 1977

\begin{tabular}{|c|c|c|}
\hline \multirow{2}{*}{ FAMILLES (pour cent) } & \multicolumn{2}{|c|}{ Secteurs } \\
\hline & 1 & 4 \\
\hline Gammaridæ & 55,4 & 41,5 \\
\hline Ancylidæe & 一 & 1,9 \\
\hline Ephemerellidæ & - & 1,9 \\
\hline $\begin{array}{ll}\text { Bætidæ } & \text { L } \\
& \text { Subi }\end{array}$ & $\begin{array}{r}32,1 \\
7,1\end{array}$ & $\frac{20,8}{-}$ \\
\hline Simuliid $\boldsymbol{x} \quad$ Ad & - & 13,2 \\
\hline $\begin{array}{r}\text { Chironomidæ } \mathrm{L} \\
\mathrm{Ny}\end{array}$ & - & $\begin{array}{l}3,8 \\
3,8\end{array}$ \\
\hline Hydroptilidæe & - & 7,5 \\
\hline Polycentropidæ & 1,8 & 5,7 \\
\hline Nymphes Trichoptères & 3,6 & 一 \\
\hline Contenus stomacaux pour 10 truites: & & \\
\hline poids sec $(\mathrm{mg})$ & 292,1 & 112,5 \\
\hline Nombre de proies & 93 & 59 \\
\hline
\end{tabular}

à 2,39 en aval. L'intensité de la consommation est encore fortement réduite chez cette espèce dans la zone boueuse.

A partir de ces deux dernières expériences on peut suivre la dégradation progressive du milieu en mai-juin qui se traduit par : une meilleure consommation en amont, une diminution générale des Mollusques et de la diversité moyenne. alors que cette période est la plus favorable au benthos de la Nivelle (NEVEU, LAPCHIN, VIGNES, 1979).

\section{DISCUSSION ET CONCLUSIONS}

Les résultats montrent que, quelles que soient l'espèce de Salmonides et l'époque, la quantité de nourriture présente dans l'estomac au même moment du cycle journalier est d'autant plus faible que le benthos est rare; c'est-à-dire que le milieu est plus colmaté par la boue.

Le colmatage du substrat réduit les interstices entre les cailloux, la circulation de l'eau et l'oxygénation. Les invertébrés ont des difficultés pour se fixer, pour circuler, les branchies se colmatent et le périphyton se développe 
mal; il y a modification de l'habitat tant au niveau chimique, physique, que trophique. II en résulte une baisse du peuple au niveau de tous les groupes rhèophiles.

Cette modification du substrat entraine non seulement une baisse du poids sec consommé de 35 à $61 \%$, mais aussi des modifications dans le choix des proies. Celles-ci sont du même type que celles observées par IVLEV (1961) sur des Cyprins. La truite commune, comme la truite arc-en-ciel, compense la diminution des stocks disponibles par une diversification des captures, ce no semble pas être le cas des juvéniles de saumon, du moins dans le cadre de cer expériences.

La modification longitudinale du substrat, en rapport avec une sédimentation abondante et régulière, est un obstacle expérimental. Elle crée une hétérogénéité supplèmentaire limitant les expériences dans le temps. L'admission d'un débit plus élevé, la création de petites crues régulières, effectuées dans des rigoles semblables, n'ont qu'une efficacité passagère; il faudrait un nettoyage régulier. On peut envisager un nettoyage avec un jet d'eau de forte puissance effectué dès le début du colmatage, mais avec destruction d'une grande partie du benthos. Le retour à des stocks benthiques normaux (en biomasse) peut alors demander plusieurs mois (MEEHAN, 1971). L'on ne connait pas non plus l'influence de telles manipulations sur les poissons, qui se trouvent alors dans des conditions de turbidité excessive.

Ce phénomène de colmatage, que l'on accélère expérimentalement dans ce genre de canaux, donne une idèe de ce qui se passe dans les cours d'eau subissant des pollutions mécaniques importantes.

CLAVEL et al (1978) trouvent une réduction de 10 à $80 \%$ de la biomasse benthique en aval d'extraction d'agrégats sur l'Allier, avec diminution de tous los groupes. En plaçant des œufs de truites dans ces zones, les mortalités embryonnaires atteignent 75 pour cent. L'étude du contenu du tube digestif de Barbeaux et de Vandoises ne montre pas de changement dans la composition; par contre, il y a augmentation du parasitisme intestinal.

Les sédiments fins limitent aussi la colonisation par la dérive. ROSENBERG et SNOW (1975), puis ROSENBERG et WIENS (1978), signalent une augmentation de la dérive sous l'influence d'un apport de particules; LUEDTKE et BRUSVEN (1976) montrent que les invertébrés ne peuvent remonter le courant faute d'une couche limite d'eau calme au niveau des substrats fins, couche dont le rôle particulier a été souligné par AMBUHL (1959.)

NUTTALL (1972), sur une rivière anglaise, trouve une réduction de la diversité benthique et une élimination de certains groupes sous l'influence des sédiments dus à l'érosion d'une partie du bassin. Il en est de mème de CHUTTER (1969) en Afrique du Sud, pour qui la faune serait surtout affectée par l'instabilité du substrat.

Les dégradations de la qualité des cours d'eau à Salmonides à la suite de coupes de bois sont un phénomène général ; l'on assiste à une augmentation des écarts de température due à l'ensoleillement, (HALL, LANTZ, 1969 ; GRAY. EDINGTON, 1969 ; BROWN, KRYGIER, 1970), mais surtout à la mise en circulation de sédiments par un développement de l'érosion. Cette sédimentation est liée principalement au débardage, à la traversée des ruisseaux, à l'accumulation de branches qui forment des barrages derrière lesquels s'accumulent les sédiments et les débris en colmatant le fond (CHAPMAN, 1962 ; HALL, LANTZ, 1969 ; 
BURNS, 1972). Dans leur ètude sur divers bassins canadiens PLAMONDON, LEPROHON et GONZALEZ (1976) trouvent dans certains cas une augmentation de 1644 pour cent pour les sédiments en suspension sans parler de l'augmentation thermique, de la teneur en minéraux, de la matière organique, le tout provoquant un développement des Sphærotilus et une chute de l'oxygène intergravellaire. WELSH, SYMONS, NARVER (1977), étudiant un petit bassin, montrent que l'exploitation de la forêt augmente de $36 \%$ les sédiments totaux et diminue de $26 \%$ le benthos, de $17 \%$ la population de Salmonides.

Or, tous ces dommages peuvent facilement être évités par la conservation d'une bande boisée d'une dizaine de mètres de part et d'autre du cours d'eau. une exploitation forestière en mosaique. l'entretien et la protection des chemins (HALL, LANTZ, 1969 ; LANTZ, 1971 ; PLAMONDON, LEPROHON. GONZALEZ. 1976).

Le développement de cultures intensives peut aussi avoir les mêmes conséquences. C'est ainsi que WELSH. SYMONS.NARVER (1977), étudiant d'autres cours d'eau de lour bassin oi s'est dévelonnce la culture do la jomme de terre trouvent une sédimentation encore plus imoortante $(+69 \%)$. associée à une contamination par pesticides. Dans ce cas le benthos est réduit de 64 pour cent, les Salmonides de 52 pour cent. Ceci montre parfaitement les dangers d'une monoculture sarclée sur les pentes d'un pavsaqe tron nivert. C'est pourquoi GUNTERMANN et al (1975), travaillant dans l'lllinois. signalent aue si le coût de l'érosion des terres agricoles passe inapercu pour le cultivateur. it neut atteindre un minimum de 10 à 15 pour cent des revenus nets nrivés de la zone aval, par les dommages causés princinalement au niveau du colmatage des réservoirs.

Le surpâturace en diminıant la converture vénétale rivulaire (caches, sunport de faune exoqène...) associé all niétinement du bétail, provoque l'érosion des berges (limonare) il neut ainsi avoir une nrande influence sur les populations de Salmonida MARCUSON (1977) troive uns réduction de $70 \%$ de la biomasse des truites communes dans cortaines rivières du Montana traversant des prairies naturelles Cette nerte est à associer à celle de $9 \%$ de la surface aqricnle utile Dar l'érosion rivulaire.

De même les rectifications do conurs d'eau peuvent avoir des conséquences néfastes dar les limons qui s'accumulent en aval. En Belnicue HUFT et TIMMERMANS (1976) trouvent ainsi des nooulations pisciaires réduites de $561 \%$ par rapport à celles hors de la zone de limonage.

Il est donc nécessairo de limiter les conséquences néfastes de tels annorts sédimentaires cuelle cri'en soit la calıse Mais s'il est relativement facile de trouver des solutions à certains de ces problèmes. il est bearıcouo plus difficile de lutter contre l'érosion de tout un bassin commo celıi de la Nivelle qui s'érode rapidement en raison de sa nature géologiculo son hydrologie et des pratiques agricoles.

\section{REFERFNCES RIP!I!OGRAPUIO!JES}

AMBOHL H. .1959. Die Bedeutung der Strömung a's Bkologischer Faktor. Schweiz Z. Hydro., 21, 133-164

BISSON P. A. DAVIS S. F. 1976 Production of iuvenile Chinook Salmon Oncorhynchus tshawytscha. in a heated models tream. Fish. Bull.. 74. 4 763-774. 
BROWN G. W., KRYGIER J. T., 1970 Effects of clear cutting on stream temperature. Water Resources Res., 6, 4, 1133-1139

BURNS J. W., 1972. Some effects of logging and associated road construction on northern California streams. Trans. Am. Fish. Soc., 101, 1, 1-17

CHAPMAN D. W., 1962. Effects of logging upon fish resources of the West Coast. J. Foresty, 60, 8, 533-537

CHUTTER F. M. 1969 The effects of silt and sand on the invertebrate fauna of streams and rivers. Hydrobiologia, 34, 1, 57-76

CLAVEL P., CUINAT R., HAMON Y., ROMANEIX C., 1978. Effets des extractions de matériaux alluvionnaires sur l'environnement aquatique dans les cours supérieurs de la Loire et de l'Allier. Bull. Fr. Pisci., 268, 123-154

CLOERN J. E., 1976. The survival of coho Salmon (Oncorhynchus kisutch) eggs in two Wisconsin tributaries of lake Michigan. Amer. Midl. Natur., 96, 2. $451-461$

E!IIIOT J. M., 1975 The growth rate of brown trout (S. trutta $L$ ) fed on reduced rations. J. Anim. Ecol., 44, 823-842

GRAY J. R. A., EDINGTON J. M., 1969. Effects of woodland clearance on stream temperature. J. Fish. Res. Bd Can., 26, 2. 399-403.

GUNTERMANN K. L., TEE M. T., SWANSON E. R., 1975. The off site sediment damage function in selected Illinois watersheds. J. Soil Water Conserv., 30. $219-224$

HALL J. D.. LANTZ R. L., 1969. Effects of logging on the habitat of Coho Salmon and Cutthroat trout in coastal streams, in T. O NORTHCOTE, Symp on Salmon and trout in streams, 1968 Univ. Bristish Columbia, 355-375.

HUET M.. TIMMERMANS I. A.. 1976. Influence sur les populations de poissons des aménagements hydrauliques de petits cours d'eau assez rapides. Travaux Stat. Rech. Groenendaal-Hœilaart, 46, D, 1-18

IVLEV V. S. 1961. Exnerimental ecolor of the feeding of fishes. New-Haven Yale Univ. Press, $300 \mathrm{p}$.

LANTZ R. L., 1971. Guidelines for stream protection in logging operations. Rep Res. Div. Oregon State Game Commission. 29 p.

LAPCHIN L., NEVEU A., sous presse. Hydrographie, climatologie et hydrologie de la Basse Nivelle, petit fleuve côtier des Pyrénées. Bull. CE.R S. Biarritz.

LUEDTKE R. J., BRUSVEN M. A.. 1976. Effects of sand sedimentation on colonization of stream insects. J. Fish. Res. Bd. Can., 33. 1881-1886

MARCUSON P. E.. 1977. The effect of cattle arazing on brown trout in Rock Creek, Montana. Montana Dept. Fish Game Fish Div.. Sp. Rep., 26 p.

MEEHAN W R.. 1971. Effects of rravel cleaninn on bottom orqanisms in three Southeast Alaska streams. Progr. Fish. Cult., 33, 2, 107-111

NEVEU A.. LAPCHIN L.. 1979. Ecologie des principaux invertébrés filtreurs de la Basse Nivelle (Pyrónées Atlantiques) I Simuliidæ (Diptera. Nematocera). Annls Limnol, 14, 3, 225-244 
NEVEU A., LAPCHIN L., VIGNES J. C., 1979. Le macrobenthos de la Basse Nivelle, petit fleuve côtier des Pyrénées Atlantiques. Ann. Zool. Ecol. Anim., $11,1,85-111$

NUTTAL P. M. 1972. The effects of sand deposition upon the macroinvertebrate fauna of the River Camel, Cornwall. Freshwat. Biol., 2, 181-186

PIHAN J., 1978. Risques climatiques d'érosion hydrique des sols en France. C. R. Colloque "Erosion des sols en milieu tempéré non méditérranéen ". Strasbourg, $20-22$ sept. 1978, 8 p.

PLAMONDON A., LEPROHON R., GONZALEZ A., 1976. Exploitation forestière et protection de quelques cours d'eau de la côte Nord. Cah. CENTREAU, $1,6,1-13$

PETERS 1. C., 1967. Effects on a trout stream of sediment from agricultural practices. J. Wild. Manag., 31, 4, 805-812

RITCHIE J. C., 1972. Sediment, fish and fish habitat. Jour. Soil. Wat. Conserv., 27, 124-125

ROSENBERG D. M., SNOW N. B., 1975. Ecological studies of aquatic organisms in the Mackenzie and Porcupine River drainages in relation to sedimentation. Dept. Env. Fish and Mar. Serv., Tech. Rept., 547, 86 p.

ROSENBERG D. M., WIENS A. P., 1978. Effects of sediment addition on macrobenthic invertebrates in a Northern Canadian River. Water. Res., 12, 753-763

SLANEY P. A., NORTHCOTE T. G., 1974. Effects of prey abundance on density and territorial behavior of young rainbow trout (S. gairdneri) in laboratory stream channels. J. Fish. Res. Bd. Can., 31, 7, 1201-1209

SYMONS P. E. K, 1968. Increase in aggression and in strength of the social hierarchy among juvenile Atlantic Salmon deprived of food. J. Fish. Res. Bd. Can., 25, 11, 2387-2401

SYMONS P. E. K., 1971. Behavioural adjustment of population density to available food by juvenile Atlantic Salmon. J. Anim. Ecol., 40, 569-587

WELCH H. E., SYMONS P. E. K., NARVER D. W., 1977. Some effects of potato farming and forest clearcutting on small New Brunswick streams. Fish. Mar. Serv. Tech. Rep., 745, 13 p. 